\title{
7 Nondeterminacy and Reasonable Choice
}

\author{
Anders Herlitz
}

Many have argued that some practical reasons admit of what could generally be called nondeterminacy: practical reasons sometimes fail to fully determine that for two comparable options, one is determinately at least as good as the other. ${ }^{1}$ The by now most common explanations of such nondeterminacy are that it is either due to vagueness (e.g., Broome 1997, 2004, 2021; Elson 2017) or because some non-conventional comparative relation such as parity obtains between two alternatives (e.g., Chang 2002; Rabinowicz 2008). Although the question of how to explain nondeterminacy has received significant attention, little has been done to identify which practical reasons admit of nondeterminacy and how they do this, i.e., which practical reasons can entail that an agent who attempts to follow and act on these reasons will face nondeterminacy problems and how these reasons entail this. This chapter hypothesizes that clues as to which practical reasons admit of nondeterminacy and how they do this can be found by studying the challenges that nondeterminacy poses for choice theory and theories of how to act rationally when practical reasons fail to fully determine what one ought to do. It argues that when otherwise acceptable decision strategies violate basic requirements of rationality because of nondeterminacy, reasons cannot admit of it, and outlines three possible explanations of how to account for this.

The chapter is structured as follows. The first section presents, in detail, nondeterminacy and a standard revision of conventional decision criteria in light of nondeterminacy. The second, third, and fourth sections introduce some challenges that nondeterminacy poses for choice theory: dynamic choice problems, underdetermination, cyclical evaluations, and violations of basic contraction consistency. The fifth section discusses some constraints that can be used with decision strategies so that these problems are avoided. In the sixth section, four views of how to understand such constraints are discussed. There is a brief concluding section. 


\section{Anders Herlitz}

\section{Nondeterminacy and Determinate Maximality}

There are good reasons to expect that practical reasons sometimes fail to fully determine what one ought to do. Here are three arguments to that effect. First, sometimes alternatives are so different from each other that it seems implausible to expect practical reasons to guide action. Are the reasons to cater to the needs of a sick relative stronger than the reasons to defend one's country against foreign occupiers? Are the reasons to finish that last work assignment on time stronger than the reasons to help one's child with their homework? Are the reasons to order the healthy salad for lunch stronger than the reasons to order the tacos that one knows will bring much more pleasure?

Second, consider the small improvement argument (see de Sousa 1974; Raz 1986; Sinnott-Armstrong 1988; Chang 2002). Consider a situation in which someone, Cleo, must choose between a career in the diplomatic corps and a career as a civil rights lawyer. Cleo recognizes many good reasons to join the diplomatic corps: it would give her a chance to promote human rights globally, serve her country, meet many interesting people, and see the world. She also recognizes many good reasons to become a civil rights lawyer: it would give her a chance to promote the rights of minorities in her community, serve her community, keep living in her hometown where she has her friends and family, and have her own family and raise children in the same loving environment that she grew up in. To Cleo, the reasons that apply to the choice do not determine that either option is better than the other. Would anything change if one of the options was improved? Would one option be clearly better than the other if it was slightly improved? Would Cleo have more reason to join the diplomatic corps than to become a civil rights lawyer if she learned that the diplomatic salary was slightly higher than she previously thought? Cleo values money and thinks that a higher salary speaks in favor of a certain career option, but would a relatively small amount of money change how the options relate to each other? To many, this seems implausible. Small improvements of one option do not tip the scale when one compares two very dissimilar options, neither of which is superior to the other. Yet, if a small improvement does not tip the scale, the initial two options cannot have been equally good. The two options must be related to each other in some other way. We can expect to find this phenomenon, what Joseph Raz (1986) once called the "mark of incommensurability," in many areas.

Third, consider a more theoretically motivated argument. It is widely recognized in population ethics that it is impossible to reconcile widely held intuitions regarding which of two populations is better; for example: "regardless of its size, a population in which everyone leads lives barely worth living is worse than a population of one billion people leading blissful lives," and "compared to a population in which everyone leads 
lives at positive welfare level $\mathrm{W}$, a population that is twice as big in which everyone leads lives that are almost as good, at positive welfare level $\mathrm{W}$-, is better" (see, e.g., Parfit 1984; Arrhenius 2000, forthcoming). Similarly, when one compares large benefits to few people with small benefits to many people, it is strikingly difficult not to end up with deeply counterintuitive conclusions, for example, that it is better to cure billions of mild headaches than to save a life (Temkin 1996, 2012; Norcross 1997; Rachels 1998). One promising way to solve these problems is by accepting the fact that reasons sometimes fail to fully determine that any option is best (Qizilbash 2007; Parfit 2016; Herlitz 2019a; Rabinowicz 2019).

Nevertheless, the aim of this chapter is not to establish that situations in which practical reasons fail to fully determine what one ought to do are possible, relevant, or common. Furthermore, I will not engage with the matter of what the best explanation for this is, whether it is sometimes indeterminate which option one has most reason to favor due to vagueness (e.g., Broome 1997), whether some alternatives are sometimes incomparable ( $\operatorname{Raz} 1986)$, whether some alternatives are sometimes "on a par" (e.g., Chang 2002), whether options are sometimes imprecisely equally as good (Parfit 2016), or whether there might be some other non-conventional comparative relation (Rabinowicz 2008). Rather, I wish to explore the following question: if these situations are relevant, how are they relevant, and under what conditions can we expect them to arise?

While the aforementioned explanations differ in some respects, they have one thing in common that is captured by the concept of nondeterminacy:

Nondeterminacy: $x$ and $y$ are nondeterminate in their ranking with respect to $p$ (i.e., that with respect to which the ranking is made) if it is not determinately true that $x$ is more $p$ than $y$, not determinately true that $x$ is less $p$ than $y$, and not determinately true that $x$ and $y$ are equally as $p$.

Nondeterminacy is irreflexive (i.e., no item is nondeterminate in its ranking with itself), symmetric (i.e., if $x$ is nondeterminate in its ranking with $y$, then $y$ is nondeterminate in its ranking with $x$ ), and non-transitive (i.e., it is not the case that if $x$ and $y$ are nondeterminate in their ranking and $y$ and $z$ are nondeterminate in their ranking, then $x$ and $z$ are necessarily nondeterminate in their ranking; see Carlson 2010). Proponents of the vagueness explanation, incomparability, parity, imprecise equality, and other non-conventional comparative relations can all agree that it is sometimes the case that some options are nondeterminate in their ranking (although they of course disagree as to why).

Nondeterminacy can actualize serious challenges to rational choice. However, it is not true, as one might initially believe, that nondeterminacy 


\section{Anders Herlitz}

undermines the very possibility of rational choice assessments in the sense that practical reasons that admit of nondeterminacy fail to give any action guidance. To see this, consider the possibility that practical reasons provide us with the following ranking of $x, y$, and $x+$ (let " $\approx$ " denote "nondeterminate in their ranking" and " $<$ " denote "determinately worse than"):

$$
x \approx y ; x+\approx y ; x<x+
$$

It is obvious that this ranking, which incorporates nondeterminacy between $x$ and $y$ and between $x+$ and $y$, does not undermine the possibility of rational choice assessments, as $x$ is not a rational choice and should not be chosen.

It is also easy enough to revise conventional decision criteria so that they can say precisely the foregoing. According to Amartya Sen, proponents of incomparability, parity, and other non-conventional comparative relations can say that only maximal options are rational, i.e., only options that are not worse than any alternative are rational (see, e.g., Sen 1970, 1997, 2017). $x$ is worse than $x+$, and thus not maximal. Proponents of the vagueness explanation of nondeterminacy can say that only options that are optimal (i.e., at least as good as every alternative) on some admissible precisification are rational choices (Fine 1975; Broome 2009). If $p$ is vague and therefore fails to fully determine a conventional ranking of $x, y$, and $x+$, one can precisify (or specify) $p$ in different ways so that a conventional ranking results. For instance, one might precisify $p$ so that it can be determined that $x$ is less $p$ than $y$ and $y$ less $p$ than $x+$. However, if $p$, which is vague, manages to fully determine that $x$ is less $p$ than $x+$, it is not admissible to precisify $p$ so that $x$ is at least as $p$ as $x+$. That is an inadmissible precisification. There is no admissible precisification on which $x$ is optimal with respect to $p$. Proponents of Wlodek Rabinowicz's version of the fitting-attitudes framework for value, which explains value relations in terms of permissible preference orderings, can say that options that are dispreferred to some alternative on all permissible preference orderings are irrational (Rabinowicz 2008). Within this fitting-attitudes framework for value, it is permissible to prefer $y$ to $x$ and to prefer $x+$ to $y$, but if it can be determined that $x$ is worse than $x+$, then $x$ is dispreferred to $x+$ on all permissible preference orderings.

To stick to the concept of nondeterminacy, I will use a more general criterion that reflects the standard ways of revising rational choice criteria within the different explanatory frameworks:

Determinate maximality (DM): An option, $x$, is determinately maxi$m a l$ with respect to $p$ if and only if (a) $x$ is not worse than any alternative with respect to $p$, and (b) it is not the case that on all admissible precisifications of $p$, there is an alternative that is better than $x .^{2}$ 
Regardless of one's view of how to explain nondeterminacy (e.g., vagueness, incomparability, parity, imprecise equality, or other non-conventional comparative relations), DM can be used to make rational choice assessments of options in choice sets in which some alternatives are nondeterminate in their ranking.

\section{More on Determinate Maximality}

One might now think that nondeterminacy problems do not pose a challenge to practical reasoning at all or that the challenge is a minor one (see, e.g., Hsieh 2007). Indeed, conventional rational choice theory cannot be used to guide action in choice situations characterized by nondeterminacy because there is no optimal alternative, but in all choice situations characterized by nondeterminacy, there is some determinately maximal alternative. So, why not just revise the decision criterion conventionally used in rational choice theory and hold that it is rational to choose any determinately maximal alternative? This section presents three arguments that challenge the plausibility of this view: it can lead to the formation of sequences of choices that are suboptimal; it neglects the fact that there might be resolutional remainder; it fails to recognize that the justificatory power grounded in comparative relations differs between situations in which some alternative is optimal and in which some alternative is determinately maximal but not optimal.

First, accepting nondeterminacy and revising one's decision criteria so that an option that is determinately maximal is rational leads to dynamic choice problems (see, e.g., McClennen 1990; Andreou 2016; Gustafsson 2016). If one adopts the view that all determinately maximal options are rational, one accepts a view that it can be permissible to form sequences of choices that are not determinately maximal (and even suboptimal), and a decision-maker who follows the strategy is exposed to a weak kind of money pump.

Consider an illustration. There are again three options: $x, x+$, and $y: x$ is nondeterminate in its ranking with $y ; x+$ is an improved version of $x$, so $x$ is worse than $x+$, but $x+$ is also nondeterminate in its ranking with $y$. In other words, we again have

$$
x \approx y ; x+\approx y ; x<x+
$$

Now, it is obvious that in a choice situation in which an agent chooses between $x, x+$, and $y, x+$ and $y$ are the only determinately maximal alternatives. According to the view that it is rational to choose any determinately maximal alternative, one of them ought to be chosen. This seems sound.

However, the approach seems less sound when one considers choice sequences. Consider the possibility that an agent will make two 
sequential choices, first $\left(\right.$ at $\left.t_{1}\right)$ a choice between $x+$ and $y$, and then $\left(\right.$ at $\left.t_{2}\right)$ a choice between sticking to the first choice or switching to $x$ :

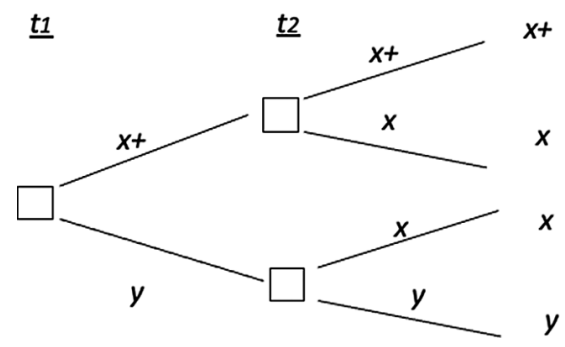

According to the view that it is rational to choose any option that is determinately maximal, it is rational to choose $y$ at $t_{1}$ and $x$ at $t_{2}$. However, this means that the agent ends up with a choice that is not determinately maximal ( $x$ is worse than $x+$, which could be chosen if the agent had chosen $x+$ at both $t_{1}$ and $t_{2}$ ), which, according to the approach that was followed, is an irrational choice. Holding all determinately maximal alternatives to be rational choices thus seems to lead to (or, more precisely, permit) irrational choices in some situations.

This phenomenon also reveals that agents who use DM as the only criterion when making choices between options that are nondeterminate in their ranking are exposed to a weak kind of money pump (Gustafsson 2016). To see this, imagine that the aforementioned sequence consisted of three sequential choices, that the agent at $t_{3}$ had the opportunity to either stick to their choice at $t_{2}$ or (if that was not their choice at this point) choose $x+$. An agent who chooses $y$ followed by $x$ would not only be rationally obliged to switch to $x+$ at $t_{3}$ but also rationally obliged to pay some money (amounting to the difference between $x$ and $x+$ ) to change their choice. If the choice sequence could be iterated, the agent would be rationally permitted to financially ruin themselves for no reason or gain whatsoever.

Adopting the view that one can make rational choices when facing options that are nondeterminate in their ranking by choosing any determinately maximal alternative means that one accepts that it can be rational to form a choice sequence that leads to an irrational choice. This is not the place to explore how often this will be a problem or the conditions under which it poses a problem. But it is obvious that it is a possibility, and this possibility alone gives us reason to question whether DM is a necessary and sufficient criterion for rational choice when some options are nondeterminate in their ranking. In the literature on dynamic inconsistency and money pumps, it has been suggested that these problems can be avoided by adopting either the view that rational agents are resolute and do not change their minds (McClennen 1990) or the view that rational agents are sophisticated choosers who use backward induction to foresee problems when they make sequential choices (Rabinowicz 1995). 
In what follows, I will suggest that dynamic choice problems should instead be solved by the introduction of some new decision criteria.

Second, it has been argued that contrary to situations in which two alternatives are optimal, when two alternatives are determinately maximal but not optimal, there is resolutional remainder:

[In choice situations characterized by nondeterminacy], there is resolutional remainder; given a list of admissible ways in which the perplexity might be resolved, there is still a further question as to how the perplexity is to be resolved, for that resolution is not simply given by arbitrarily opting for one admissible resolution over another.

(Chang 2002: 684-685)

In some choices between options that are nondeterminate in their ranking, it seems as though something is unresolved if one merely picks an option that is determinately maximal. To take this intuition seriously, this something ought to be addressed by practical reasoning in some way.

Third, there is a difference between what might be called the justificatory power of an option being optimal and an option being determinately maximal but not optimal. When two options are (determinately) equally good, this provides a sufficient justification for an agent to choose either one of them. When neither of two options is (determinately) at least as good as the other, this might provide some justification for an agent to choose either one of them, but this justification seems weaker. In other words, it is not obvious that the justification of a choice that is grounded in how alternatives relate to each other is sufficiently strong to meet the requirements of rationality when an option is merely determinately maximal but not optimal, as opposed to when an option is optimal (i.e., at least as good as all alternatives).

\section{Introducing New Criteria}

A different way of responding to nondeterminacy problems is to say that when two options are nondeterminate in their ranking with respect to some decision criterion or reason, some other criterion or reason should be used to rank them so that one can establish a conventional ranking of all pairs of options. This approach clearly deals with potential resolutional remainder and, insofar as it can determine an option that is at least as good as all alternatives, it leads to a strong justification.

For instance, someone may propose the idea that when some set $P$ of practical reasons that apply to the choice admits of nondeterminacy, one ought to make pairwise comparisons of the options that are nondeterminate in their ranking by ranking them with respect to some other reason, $Q$, that does not admit of nondeterminacy. In other words:

Imposing conventional pairwise rankings: $x$ is worse than $y$ if and only if $x$ is worse than $y$ with respect to the set of primary practical 
reasons $P$, or $x$ and $y$ are nondeterminate in their ranking with respect to $P$ and $x$ is worse than $y$ with respect to secondary reason $Q$.

At a general level, and insofar as one does not qualify what $Q$ might be and how it might relate to $P$, this view is problematic. To see this, consider the following possibility. Assume, again, that with respect to $P, x$, $x+$, and $y$ relate to each other in the following way:

$$
x \approx y ; x+\approx y ; x<x+
$$

If one does not qualify $Q$ in any way, it is perfectly possible that with respect to $Q, x, x+$, and $y$ relate to each other in the following way:

$$
x+<y ; y<x ; x+<x
$$

Imposing conventional pairwise rankings then can generate the following cyclical ranking of the options:

$$
x+<y ; y<x ; x<x+
$$

Thus, $x+$ is worse than $y$ because $x+$ and $y$ are nondeterminate in their ranking with respect to $P$, and $x+$ is worse than $y$ with respect to $Q ; y$ is worse than $x$ because $y$ and $x$ are nondeterminate in their ranking with respect to $P$, and $y$ is worse than $x$ with respect to $Q$, and $x$ is worse than $x+$ because $x$ is worse than $x+$ with respect to $P$. According to this approach it is then irrelevant how $x$ and $x+$ relate to each other with respect to $Q$.

This is not just a theoretical possibility. To see this, consider an illustration. Imagine that $x, x+$, and $y$ are different wines and that $P$ reflects the set of reasons that underlie how much one will enjoy them. Let $Q$ reflect the reason to favor more environmentally friendly wines, something an agent could plausibly use as a criterion to establish pairwise rankings of alternative wines that are nondeterminate in their ranking with respect to how much she will enjoy them. There is no obvious and necessary relation between how much one will enjoy a wine and how environmentally friendly the production of the wine is. Therefore, it is perfectly possible that insofar as how much one will enjoy a wine admits of nondeterminacy, imposing conventional pairwise rankings of wines by looking at how environmentally friendly the production is can lead to cyclical evaluations. Assume that $x$ is a white wine (say a Pinot Grigio) that is nondeterminate in its ranking with red wine $y$ (say a Bordeaux) with respect to how enjoyable it is to consume and that $x+$ is a slightly better white wine (say a Chablis) that is also nondeterminate in its ranking with red wine $y$. As it happens, the production process of $x$ is the most environmentally friendly, followed by the production process of $y$, with the production process of $x+$ being the least environmentally friendly. An agent who 
makes pairwise rankings of the wines by determining that a wine is worse than another if and only if it is less enjoyable, or if it is nondeterminate in its ranking with the other wine but its production process is less environmentally friendly, will generate a cyclical ranking of the wines.

It is sometimes suggested that one can avoid accepting unpalatable rankings such as these by individuating alternatives in different ways depending on the comparative context (see Broome 1991; Arrhenius 2009; Voorhoeve 2014; Herlitz 2019b, 2020a). If one, for instance, individuated $y$ in different ways depending on whether one compares $y$ with $x$ (say $y^{*}$ ) or with $x+\left(\right.$ say $\left.y^{* *}\right)$, the ranking would no longer be cyclical. The following is not a cyclical ranking of the options:

$$
x+<y * * ; y *<x ; x<x+
$$

Although in principle a theoretically possible way to refute the argument, this solution is not appealing. As John Broome (1991) has pointed out, individuating alternatives in different ways is always possible, but because of this, one must have a good reason to change the individuation. In general, alternatives ought to be individuated in such a way that all features that are pertinent in light of what matters to the comparison are included in the individuation (Broome 1991; Voorhoeve 2014; Herlitz $2019 \mathrm{~b}, 2020 \mathrm{a})$. If I wish to evaluate two wines in light of how enjoyable they are, I ought to individuate them so that their enjoyable properties are included. In the previous example, it is obvious that there is a way of individuating the alternatives so that both the features pertinent in light of $P$ and the features pertinent in light of $Q$ are included and generate a cyclical ranking. For instance, one can individuate the three wines in a way that includes both their enjoyable features and facts about how environmentally friendly their production processes are and end up with a cyclical ranking of the wines. This suffices for the argument that imposing conventional pairwise rankings can lead to cyclical rankings.

What started as a nondeterminacy problem - a problem relating to the fact that reasons fail to determine an option that is at least as good as all alternatives - has now turned into a cyclical ranking of the options, with all options being worse than some alternative (see Herlitz 2020b). ${ }^{4}$ Although acyclicity is not necessary for rational choice (see, e.g., Schwartz 1986; Duggan 2013; Herlitz 2020c), cyclical orderings are generally seen as a bigger problem than nondeterminacy (see, e.g., Broome 2004).

Furthermore, it is questionable whether the methods that can be used to identify rational options when one deals with cyclical rankings generate plausible results in this case. Conventional ways of dealing with cycles (e.g., referring to Schwartz sets or uncovered options) would say that all options are equally rational choices in the previous example. This seems questionable. If $x, x+$, and $y$ are three wines, one is primarily interested in how much one will enjoy the wines, and $x$ is determinately less enjoyable than $x+$, it is questionable whether it is rational to choose $x$ (see Herlitz 2020b). 


\section{Anders Herlitz}

\section{Two-step Models}

Rather than introducing a new reason in order to determine how options relate to each other in pairwise comparisons, one might be attracted by the idea of introducing a new reason in order to rank the options that remain after DM has been applied. After all, it seems implausible to consider $x$ a viable option at all if it is determinately true that $x$ is worse than $x+$ with respect to what matters to the choice. Also, this approach clearly deals with potential resolutional remainder, and since it determines an option that is at least as good as all alternatives, it leads to as strong a justification as the one provided in cases in which there is no nondeterminacy.

In other words, one might propose the following idea:

Two-step model: Choose an option that is determinately maximal with respect to $P$. If more than one option is determinately maximal with respect to $P$, choose an option in this set that is at least as good as all alternatives in the set with respect to $Q$.

This has been a somewhat popular move among those who recognize nondeterminacy problems and take them seriously. Chang (2013) has famously argued that when what she calls "given reasons" fail to fully determine what one ought to do, one ought to introduce a created reason grounded in the agent's volition - and do what is best with respect to the created reason. Norman Daniels (2008) has argued that substantive principles used to rank different healthcare policies admit of nondeterminacy and fail to fully determine what policymakers ought to do; in those situations, he argues that one ought to resort to a deliberative process and rank alternatives with respect to the criteria that transpire from such a process. Fröding and Peterson (2012) have suggested that a virtuous agent might face options that are nondeterminate in their ranking with respect to moral reasons and that the virtuous agent will compare these options in terms of how good they are with respect to relevant non-moral reasons. I myself have advocated such two-step models in the context of allocating scarce health-related resources, in social choice situations, and in the context of clinical choice situations, person-centered care, and shared decision-making (Herlitz 2017, 2018, forthcoming; Herlitz \& Sadek 2021).

However, this method violates what many (see, e.g., Chernoff 1954; Sen 1970; Fleurbaey, Tungodden and Vallentyne 2009) take to be a basic requirement of rationality, i.e., the so-called basic contraction consistency, sometimes called property alpha (see Herlitz 2019b):

Basic contraction consistency: If an alternative, $x$, is permissible in a choice set $\mathrm{C}$, then it is also permissible in a choice set C' that is a subset of $\mathrm{C}$ and contains $x$. 
It can be easily seen that basic contraction consistency is violated by the two-step model. Consider once again $x, x+$, and $y$, which relate to each other with respect to $P$ in the following way:

$$
x \approx y ; x+\approx y ; x<x+
$$

and which relate to each other with respect to $Q$ in the following way:

$$
x+<y ; y<x ; x+<x
$$

According to the two-step model, $y$ should be chosen (and is the only permissible option) if the choice set is $\{x, x+, y\}$. This is because only $x+$ and $y$ are determinately maximal with respect to $P$, and $x+$ is worse than $y$ with respect to $Q$. Since $x$ is worse than $x+$ with respect to $P$, it is not determinately maximal and is therefore discarded in the first step; it is irrelevant how $x$ and $x+$ relate to each other with respect to $Q$.

However, $x$ should be chosen (and is the only permissible option) according to the two-step model if the choice set is $\{x, y\}$. Both $x$ and $y$ are determinately maximal with respect to $P$ when $x+$ is not in the choice set, and $y$ is worse than $x$ with respect to $Q$. Since $x+$ is not in the choice set, there is no option that is better than $x$ with respect to $P$ in the choice set; there are no grounds for ruling out $x$ in the first step, so how good $x$ is with respect to $Q$ becomes highly relevant.

Obviously, $\{x, y\}$ is a subset of $\{x, x+, y\}$, and whereas $y$ is permissible in $\{x, x+, y\}$, it is not permissible in $\{x, y\}$, which contains $y$. In other words, basic contraction consistency is violated.

The two-step model would violate basic contraction consistency if $x, x+$, and $y$ are the three wines; $P$ reflects how enjoyable they are; and $Q$ reflects how environmentally friendly the production processes of the wines are, as described in the previous section. When choosing between all three wines, the approach suggests that one first discards the worse white wine, $x$, and then ranks the better white wine, $x+$, and the red wine, $y$, with respect to how environmentally friendly their production processes are. Since the production process of the red wine is more environmentally friendly than that of the better white wine, the approach suggests that one will choose the red wine. When choosing only between the less enjoyable white wine and the red wine, all options are nondeterminate in their ranking with respect to how enjoyable they are, so all options are evaluated in terms of how environmentally friendly their production processes are. Since the production process of the less enjoyable white wine is more environmentally friendly than that of the red wine, the approach suggests that one ought to choose the more environmentally friendly red wine.

\section{Avoiding Violations of Rationality Requirements}

The previous sections reveal that nondeterminacy in principle can cause agents who adopt seemingly attractive and plausible decision strategies 


\section{Anders Herlitz}

to violate what appear to be basic requirements of rationality. An agent who adopts the strategy "choose any determinately maximal alternative" might form choice sequences that lead to choices that are not determinately maximal. An agent who attempts to impose conventional comparative relations on pairs of options that are nondeterminate in their ranking with respect to the reasons that apply to the situation by invoking some new reason might end up generating cyclical evaluations of her options. An agent who discards alternatives that are not determinately maximal and compares the remaining options that are determinately maximal with respect to some new reason might violate basic contraction consistency. What can we learn from this?

First, it can be noted that both the argument that illustrated how nondeterminacy can spawn cyclical evaluations and the argument that illustrated how two-step models violate basic contraction consistency rely on an important assumption. For these arguments to work, it must be assumed that the reasons that admit of nondeterminacy and the reason that is brought in to establish a ranking do not relate to each other in specific ways.

If one, by contrast, puts constraints on what might qualify as a valid reason that plays this role, the arguments can be refuted (see Herlitz 2019b). As an illustration, consider again the case in which $x, x+$, and $y$ are three wines, and there is nondeterminacy in their ranking when one ranks them with respect to how enjoyable, $P$, they are $(x \approx y$ and $x+\approx y)$. Assume further that the two white wines, $x$ and $x+$, are enjoyable because they are light and refreshing, and the red wine, $y$, is enjoyable because it has a rich taste. Enjoyable, $P$, admits of nondeterminacy because both wines that are light and refreshing and wines with a rich taste are enjoyable, but it is not fully determined how these features relate to each other for overall assessments of how enjoyable different wines are. Insofar as the reason one uses to establish conventional rankings or to choose between options in a second step, $Q$, is entirely unrelated to how enjoyable the wines are, for instance, if this reason relates to how environmentally friendly the production processes are, imposing conventional comparative relations and the two-step model run into problems (as illustrated in the previous sections). By contrast, if one holds that the reason used to establish a conventional ranking must be a reason, $Q^{*}$, that determines how being light and refreshing and being rich in taste relate to each other with respect to how the features contribute to overall assessments of how enjoyable different wines are, one can avoid these problems. For instance, $Q$ * could be "light and refreshing wines are better than wines with a rich taste," or "wines with a rich taste are better than light and refreshing wines." Such a reason, $Q *$, will generate rankings of the less enjoyable white wine and the red wine as well as of the more enjoyable white wine and the red wine such that if it determines that the less enjoyable white wine is better than the red wine, then also the more enjoyable white wine will be better than the red wine, and if the more enjoyable white wine is worse than the red wine, then also the less enjoyable white wine will be worse than the red 
wine. It follows from the constraints on $Q^{*}$ that if $y<x$ with respect to $Q^{*}$, then $y<x+$ with respect to $Q^{*}$, and if $x+<y$ with respect to $Q^{*}$, then $x<y$ with respect to $Q^{*}$. This means that there will be no cycle in the ranking if one imposes conventional comparative rankings with reference to $Q^{*}$ and relying on $Q^{*}$ in a two-step model will not lead to violations of basic contraction consistency.

The point can be generalized within specific explanatory frameworks of nondeterminacy. Consider, for instance, how one can impose constraints on $Q$ with reference to the supervaluationist approach to vagueness within the framework that holds that nondeterminacy is due to vagueness. Assume that $x$ and $y$ are nondeterminate in their ranking with respect to $P$ and that $x+$ and $y$ are nondeterminate in their ranking with respect to $P$ because $P$ is vague so that it is indeterminate whether $x$ is more $P$ than, less $P$ than, or equally as $P$ as $y$ and indeterminate whether $x+$ is more $P$ than, less $P$ than, or equally as $P$ as $y$. According to the supervaluationist approach to vagueness, a sentence that contains a vague term is "supertrue" if it is true on all admissible precisifications and "superfalse" if it is false on all admissible precisifications (Fine 1975; Broome 2009; Andersson 2017). An admissible precisification is one that is in accordance with ordinary language and that respects so-called penumbral truths (Fine 1975). If one holds that $Q$ must be such that it provides an admissible precisification of $P$, imposing conventional comparative rankings will not generate cyclical rankings, and the two-step model will not violate basic contraction consistency. This is because introducing and relying on a $Q$ that would lead to cyclical rankings or violations of basic contraction consistency would mean that one precisifies $P$ in a way that contradicts penumbral truths, which is inadmissible.

Insofar as one commits to using the same $Q$ that provides an admissible precisification of $P$ to determine a ranking of the alternatives in all situations in which some alternatives are indeterminate in their ranking with respect to $P$ (which is vague), one also avoids the risk of forming sequences of choices that lead to options that are determinately worse (with respect to $P$ ) than some available choice sequence that is available. If an agent commits to determine what to do when the options are indeterminate in their ranking with respect to $P$ by ranking the options with respect to $Q$ and if $Q$ provides an admissible precisification of $P$, then if $x+<y$ at $t_{1}$ then $x<y$ at $t_{2}$. An agent who commits to using the same $Q$ that provides an admissible precisification of $P$ to determine a ranking of the alternatives will, in other words, either choose $x+$ at $t_{1}$ and $x+$ at $t_{2}$ or $y$ at $t_{1}$ and $y$ at $t_{2}$, neither of which leads to a choice that is determinately worse with respect to $P$ than any available alternative.

It is obvious from the aforementioned that one can avoid the problems outlined in the previous sections of this chapter if one accepts that nondeterminacy is due to vagueness and imposes constraints on what qualifies as an admissible $Q$ in terms of admissible precisifications. Somewhat similarly, proponents of the fitting-attitudes approach to value can within 


\section{Anders Herlitz}

their framework impose constraints on what is an admissible $Q$ in terms of permissible preference orderings. For instance:

Yields permissible preference orderings over the choice set: $Q$ is admissible if and only if it yields preference orderings over a choice set that are permissible given the rankings established by $P$.

(see Rabinowicz 2008; Herlitz 2019b)

In other words, it is noteworthy that, given certain explanations of nondeterminacy, there are views of how to make rational choices when reasons fail to fully determine a best alternative by introducing new reasons to establish conventional rankings that avoid the problems outlined in the previous section. These views say that one must impose constraints on what the new reasons that establish conventional rankings might be like, somewhat similar to how supervaluationist approaches to vagueness impose constraints on what constitutes admissible precisifications of vague terms.

\section{Understanding How Reasons Cannot Admit of Violations of Rationality Requirements}

Given that one can avoid choice-theoretical challenges by putting constraints on reasons that are introduced to create conventional rankings of options that are nondeterminate in their ranking, one can perhaps learn something about when and how nondeterminacy occurs by studying how decision strategies might violate rationality requirements. It is possible that reasons cannot admit of nondeterminacy in such a way that doing what is permissible according to these reasons can mean that one violates basic requirements of rationality. This section explores this possibility.

Consider four views of the problems introduced in the previous sections. First, it is of course possible to refute the relevance of the arguments:

The accept irrationality view: Reasons can admit of nondeterminacy, and this means that acceptable decision strategies sometimes lead to violations of basic requirements of rationality. However, this is not a problem.

Although this is certainly a possible position, I believe most people would favor views that do not entail cyclical rankings or the violation of basic contraction consistency.

Here are three views that achieve this:

The properties of nondeterminacy view: Reasons can admit of nondeterminacy, but the phenomenon - nondeterminacy - has certain 
properties that constrain what it means to respond appropriately to these reasons.

On this view, nondeterminacy has certain properties with substantive implications for how it is appropriate to respond to the reasons that admit of nondeterminacy when some options are nondeterminate in their ranking. The relation " $x$ is at least as good as $y$ " has a property that implies that it is appropriate to choose $x$. By contrast, the relation " $x$ is nondeterminate in its ranking with $y$ " might have a property that implies that it is appropriate to use a certain method to choose between $x$ and $y$, and this method has formal constraints in line with the constraints discussed in the previous section.

On the properties of the nondeterminacy view, the features of nondeterminacy explain that one ought to respond to nondeterminacy in a way that avoids violating basic requirements of rationality. The nondeterminacy phenomenon as such constrains rational agents that face it.

The not all reasons view: Reasons can admit of nondeterminacy, but the domain of valid reasons cannot be such that responding to the reasons means that one violates basic requirements of rationality.

On this view, the domain of valid reasons has certain properties such that if the valid first-order reasons admit of nondeterminacy, the only second-order reasons that might be used to establish conventional rankings that are valid are reasons that do not lead to violations of basic requirements of rationality. On this view, basing one's choice of wine - in the example used above on how environmentally friendly the production process is amounts to basing one's choice of wine on a second-order reason that is invalid.

On the not all reasons view, the domain of valid reasons is such that the only second-order reasons that are valid are those that do not lead to violations of basic requirements of rationality, which explains how one ought to respond to nondeterminacy in a way that avoids violations of basic requirements of rationality. The domain of valid reasons ensures that rational agents that face nondeterminacy can never act on valid reasons and violate rationality requirements because the domain of valid reasons has an architecture that renders this impossible.

The rationality obliges view: Reasons can admit of nondeterminacy, but for an agent to be rational, she must adopt a decision strategy that does not violate basic requirements of rationality.

On this view, rationality has certain properties that constrain how one can rationally respond to nondeterminacy. Accordingly, choosing a wine in the recurrent example with reference to the environmental friendliness of the production process is irrational because norms of rationality 


\section{Anders Herlitz}

require that rational agents avoid decision strategies that violate basic requirements of rationality. On the rationality obliges view, that this is what it means to be rational explains how one ought to respond to nondeterminacy in a way that avoids violating basic requirements of rationality. Rationality thus constrains agents who face nondeterminacy.

The properties of the nondeterminacy view, the not all reasons view, and the rationality obliges view can all explain why a rational agent who acts on valid reasons that admit of nondeterminacy will avoid violating basic requirements of rationality. As such, each of these views offers a solution to the choice-theoretical challenges that arise once one accepts the possibility of nondeterminacy. However, the views explain how rational agents avoid these problems in different ways, and these different ways have very different implications for different areas of interest to decision theorists and philosophers. Those who accept the properties of the nondeterminacy view will have to accept that comparative relations carry far more information than is often thought and that they also can have wide-ranging implications for how rational agents are permitted to make choices. Those who accept the not all reasons view will have to accept that there are formal constraints on the domain of valid reasons that are indirectly inferred from constellations in the domain of reason in complex ways. Finally, those who accept the rationality obliges view will have to accept a view of rationality that is rich enough to have implications that impose constraints on second-order reasons.

\section{Conclusion}

In this chapter, I have outlined some choice-theoretical challenges that arise if one accepts the possibility of nondeterminacy, i.e., that actionguiding reasons may fail to fully determine a best option. I demonstrated that nondeterminacy can lead agents to form suboptimal choice sequences and that if they use seemingly plausible decision strategies, they might make cyclical rankings of their options or violate basic contraction consistency. I also demonstrated that there are ways to impose constraints on decision strategies in order to avoid these problems but showed that these constraints can be derived either from the phenomenon of nondeterminacy, from the reasons themselves, or from norms of rationality. Whichever way one attempts to derive the constraints, there will be implications for the phenomenon of nondeterminacy, for what the domain of reasons is like, or for norms of rationality.

\section{Notes}

1 For helpful comments and exchanges on an earlier version, I thank Henrik Andersson, Chrisoula Andreou, Gustaf Arrhenius, John Broome, Krister Bykvist, Ruth Chang, Luke Elson, Mozaffar Qizilbash, Miriam 
Schoenfield, Wlodek Rabinowicz and other participants at the workshop 'Incommensurability: Vagueness, Parity and Other Non-Conventional Value Relations' at the Institute for Futures Studies, Stockholm in December 2019. I am very grateful for additional feedback on a later draft from Henrik Andersson and Wlodek Rabinowicz. Research for this paper was supported by the Swedish Research Council (2017-01382) and Riksbankens Jubileumsfond (M17-0372:1).

2 Within the fitting-attitudes framework for value, saying that $x$ is not worse than any alternative with respect to $p$ amounts to saying that there is no alternative, $z$, such that it is required to disprefer $x$ to $z$.

3 When Ruth Chang first introduced this idea, she tied it to parity, her favored explanation of nondeterminacy. However, there is no reason to tie the phenomenon of resolutional remainder to a particular explanation of nondeterminacy (Wasserman 2004).

4 Somewhat relatedly, insofar as nondeterminacy is possible, it is in principle possible that it occurs when one applies one of several decision criteria.

\section{References}

Andersson, H. (2017), How It All Relates: Exploring the Space of Value Comparisons (Department of Philosophy, Lund University).

Andreou, C. (2016), ‘Dynamic Choice', in E.Zalta (ed.), The Stanford Encyclopedia of Philosophy (Center for the Study of Language and Information Stanford University).

Arrhenius, G. (2000), 'An Impossibility Theorem for Welfare Axiologies', Economics and Philosophy 16/2: 247-266.

Arrhenius, G. (2009), 'Can the Person Affecting Restriction Solve the Problems in Population Ethics?', in M. A. Roberts and D. T. Wasserman (eds.), Harming Future Persons: Ethics, Genetics and the Nonidentity Problem (Springer), 289-314.

Arrhenius, G. (forthcoming). Population Ethics: The Challenge of Future Generations.

Broome, J. (1991), Weighing Goods (Blackwell).

Broome, J. (1997), 'Is Incommensurability Vagueness?', in R. Chang (ed.), Incommensurability, Incomparability, and Practical Reason (Harvard University Press), 67-89.

Broome, J. (2004), Weighing Lives (Oxford University Press).

Broome, J. (2009), 'Reply to Rabinowicz', Philosophical Issues 19/1: 412-417.

Broome, J. (2021), 'Incommensurability Is Vagueness', in H. Andersson and A. Herlitz (eds.), Value Incommensurability: Ethics, Risk and Decision-Making (Routledge).

Carlson, E. (2010), 'Parity Demystified', Theoria 76/2: 119-128.

Chang, R. (2002), 'The Possibility of Parity', Ethics 112/2: 659-688.

Chang, R. (2013), 'Grounding Practical Normativity: Going Hybrid', Philosophical Studies 164: 163-187.

Chernoff, H. (1954), 'Rational Selection of Decision Functions', Econometrica 22/4: 422-443.

Daniels, N. (2008), Just Health: Meeting Health Needs Fairly (Cambridge University Press).

Duggan, J. (2013), 'Uncovered Sets', Social Choice and Welfare 41: 489-535. 


\section{Anders Herlitz}

Elson, L. (2017), 'Incommensurability as Vagueness: A Burden-Shifting Argument', Theoria 83/4: 341-363.

Fine, K. (1975), 'Vagueness, Truth and Logic', Synthese 30: 265-300.

Fleurbaey, M., Tungodden, B., and Vallentyne, P. (2009), 'On the Possibility of Nonaggregative Priority for the Worst Off', Social Philosophy and Policy 26/1: 258-285.

Fröding, B., and Peterson, M. (2012), 'Virtuous Choice and Parity', Ethical Theory and Moral Practice 15: 71-82.

Gustafsson, J. (2016), 'Money Pumps, Incompleteness, and Indeterminacy', Philosophy and Phenomenological Research 92/1: 60-72.

Herlitz, A. (2017), 'Comparativism and the Grounds for Person-Centered Care and Shared Decision Making', Journal of Clinical Ethics 28/4: 269-278.

Herlitz, A. (2018), 'Committing to Priorities: Incompleteness in Macro-Level Health Care Allocation and its Implications', Journal of Medicine and Philosophy 43/6: 724-745.

Herlitz, A. (2019a), 'Nondeterminacy and Population Ethics', in P. Bowman and K. B. Rasmussen (eds.), Studies on Climate Ethics and Future Generations, Working paper series 2019: 1-11 (Institute for Futures Studies).

Herlitz, A. (2019b), 'Nondeterminacy, Two-Step Models, and Justified Choice', Ethics 129/2: 284-308.

Herlitz, A. (2020a), 'Stable and Unstable Choices', Economics and Philosophy 36/1: 113-125.

Herlitz, A. (2020b), 'Nondeterminacy, Cycles and Rational Choice', Analysis 80/3: 443-449.

Herlitz, A. (2020c), 'Non-transitive Better than Relations and Rational Choice', Philosophia 48: 179-189.

Herlitz, A. (Forthcoming), 'Toward a Hybrid Theory of How to Allocate HealthRelated Resources', Journal of Medicine and Philosophy.

Herlitz, A., and Sadek, K. (2021), 'Social Choice, Nondeterminacy, and Practical Reasoning', Res Philosophica 98/3: 1-25.

Hsieh, N.-H. (2007), 'Is Incomparability a Problem for Anyone?', Economics and Philosophy 23/1: 65-80.

McClennen, E. F. (1990), Rationality and Dynamic Choice: Foundational Explorations (Cambridge University Press).

Norcross, A. (1997), 'Comparing Harms: Headaches and Human Lives', Philosophy and Public Affairs 26/2: 135-167.

Parfit, D. (1984), Reasons and Persons (Clarendon Press).

Parfit, D. (2016), 'Can We Avoid the Repugnant Conclusion?', Theoria 82/2: 110-127.

Qizilbash, M. (2007), 'The Mere Addition Paradox, Parity and Vagueness', Philosophy and Phenomenological Research 75/1: 129-151.

Rabinowicz, W. (1995), 'To Have One's Cake and Eat It, Too: Sequential Choice and Expected-Utility Violations', Journal of Philosophy 92/11: 586-620.

Rabinowicz, W. (2008), 'Value Relations', Theoria 74/1: 18-49.

Rabinowicz, W. (2019), 'Can Parfit's Appeal to Incommensurabilities Block the Continuum Argument for the Repugnant Conclusion?', in P. Bowman and K. B. Rasmussen (eds.), Studies on Climate Ethics and Future Generations, Working paper series 2019: 1-11 (Institute for Futures Studies). 
Rachels, S. (1998), 'Counterexamples to the Transitivity of Better Than', Australasian Journal of Philosophy 76/1: 71-83.

Raz, J. (1986), The Morality of Freedom (Oxford University Press).

Schwartz, T. (1986), The Logic of Collective Choice (Columbia University Press). Sen, A. (1970), Collective Choice and Social Welfare (Holden-Day).

Sen, A. (1997), 'Maximization and the Act of Choice', Econometrica 65/4: $745-779$.

Sen, A. (2017), 'Reason and Justice: The Optimal and the Maximal', Philosophy 92/1: 5-19.

Sinnott-Armstrong, W. (1988), Moral Dilemmas (Blackwell).

de Sousa, R. (1974), 'The Good and the True', Mind 83/332: 534-551.

Temkin, L. (1996), 'A Continuum Argument for Intransitivity', Philosophy and Public Affairs 25/3: 175-210.

Temkin, L. (2012), Rethinking the Good: Moral Ideals and the Nature of Practical Reasoning (Oxford University Press).

Voorhoeve, A. (2014), 'How Should We Aggregate Competing Claims?', Ethics 125/1: 64-87.

Wasserman, R. (2004), 'Indeterminacy, Ignorance and the Possibility of Parity', Philosophical Perspectives 18/1: 391-403. 